\title{
The Trace Anomaly and Dynamical Vacuum Energy in Cosmology
}

\author{
Emil Mottola \\ Theoretical Division, Los Alamos National Laboratory \\ Los Alamos, NM 87545 USA \\ and \\ Theoretical Physics Group, PH-TH, CERN \\ CH-1211, Geneva 23, Switzerland \\ E-mail: emil@lanl.gov and emil.mottola@cern.ch \\ Invited Talk at the Ninth Conference on \\ Quantum Field Theory under the Influence of External Conditions \\ The University of Oklahoma, Norman, OK \\ September 21-25, 2009 \\ To appear in the Proceedings of QFEXT09
}

The trace anomaly of conformal matter implies the existence of massless scalar poles in physical amplitudes involving the stress-energy tensor. These poles may be described by a local effective action with massless scalar fields, which couple to classical sources, contribute to gravitational scattering processes, and can have long range gravitational effects at macroscopic scales. In an effective field theory approach, the effective action of the anomaly is an infrared relevant term that should be added to the Einstein-Hilbert action of classical General Relativity to take account of macroscopic quantum effects. The additional scalar degrees of freedom contained in this effective action may be understood as responsible for both the Casimir effect in flat spacetime and large quantum backreaction effects at the horizon scale of cosmological spacetimes. These effects of the trace anomaly imply that the cosmological vacuum energy is dynamical, and its value depends on macroscopic boundary conditions at the cosmological horizon scale, rather than sensitivity to the extreme ultraviolet Planck scale. 


\section{THE COSMOLOGICAL CONSTANT AND VACUUM ENERGY}

In classical General Relativity, the requirement that the field eqs. involve no more than two derivatives of the metric tensor allows for the possible addition of a constant term, the cosmological term $\Lambda$, to Einstein's eqs.

$$
R_{\nu}^{\mu}-\frac{R}{2} \delta_{\nu}^{\mu}+\Lambda \delta_{\nu}^{\mu}=\frac{8 \pi G}{c^{4}} T_{\nu}^{\mu}
$$

If transposed to the right side of this relation, the $\Lambda$ term corresponds to a constant energy density $\rho_{\Lambda}=c^{4} \Lambda / 8 \pi G$ and isotropic pressure $p_{\Lambda}=-c^{4} \Lambda / 8 \pi G$ permeating all of space uniformly, and independently of any localized matter sources. Hence, even if the matter stress tensor $T_{\nu}^{\mu}=0$, a cosmological term causes spacetime to become curved with a radius of curvature of order $|\Lambda|^{-\frac{1}{2}}$. In purely classical physics there is no natural scale for $\Lambda$. Indeed if $\hbar=0$ and $\Lambda=0$, there is no fixed length scale at all in the vacuum Einstein equations, $G / c^{4}$ being simply a conversion factor between the units of energy and those of length. Hence $\Lambda$ may take on any value whatsoever with no difficulty (and with no explanation) in classical General Relativity.

As soon as we allow $\hbar \neq 0$, there is a quantity with the dimensions of length that can be formed from $\hbar, G$, and $c$, namely the Planck length,

$$
L_{p l} \equiv\left(\frac{\hbar G}{c^{3}}\right)^{\frac{1}{2}}=1.616 \times 10^{-33} \mathrm{~cm} .
$$

Hence when quantum theory is considered in a general relativistic setting, the quantity,

$$
\lambda \equiv \Lambda L_{p l}^{2}=\frac{\hbar G \Lambda}{c^{3}}
$$

becomes a dimensionless pure number, whose value one might expect a theory of gravity incorporating quantum effects to address.

Some eighty years ago W. Pauli was apparently the first to consider the question of the effects of quantum vacuum fluctuations on the the curvature of space [1]. Pauli recognized that the sum of zero point energies of the two transverse electromagnetic field modes in vacuo

$$
\rho_{\Lambda}=2 \int^{L_{\min }^{-1}} \frac{d^{3} \vec{k}}{(2 \pi)^{3}} \frac{\hbar \omega_{k}}{2}=\frac{1}{8 \pi^{2}} \frac{\hbar c}{L_{m i n}^{4}}=-p_{\Lambda}
$$

contribute to the stress-energy tensor of Einstein's theory as would an effective cosmological term $\Lambda>0$. Since the integral $(1.4)$ is quartically divergent, an ultraviolet cutoff $L_{\min }^{-1}$ of $(1.4)$ at large 
$|\vec{k}|$ is needed. Taking this short distance cutoff $L_{\text {min }}$ to be of the order of the classical electron radius $e^{2} / m c^{2}$, Pauli concluded that if this estimate were correct, Einstein's theory with this large a $\Lambda$ would lead to a universe so curved that its total size "could not even reach to the moon." If instead of the classical electron radius, the apparently natural but much shorter length scale of $L_{\min } \sim L_{p l}$ is used to cut off the frequency sum in $(1.4)$, then the estimate for the cosmological term in Einstein's equations becomes vastly larger, and the entire universe would be limited in size to the microscopic scale of $L_{p l}(1.2)$ itself, in even more striking disagreement with observation.

Clearly the naive estimate of the contribution of short distance modes of the electromagnetic field to the curvature of space, by using (1.4) as a source for Einstein's eqs. (1.1) is not correct. The question is why. Here the Casimir effect may have something to teach us. The vacuum zero point fluctuations being considered in (1.4) are the same ones that contribute to the Casimir effect, but this estimate of the scale of vacuum zero point energy, quartically dependent on a short distance cutoff $L_{m i n}$, is certainly not relevant for the effect observed in the laboratory [2]. In calculations of the Casimir force between conductors, one subtracts the zero point energy of the electromagnetic field in an infinitely extended vacuum (with the conductors absent) from the modified zero point energies in the presence of the conductors. It is this subtracted zero point energy of the electromagnetic vacuum, depending upon the boundary conditions imposed by the conducting surfaces, which leads to experimentally well verified results for the force between the conductors.

In the renormalization procedure the ultraviolet cutoff $L_{\text {min }}^{-1}$ drops out, and the distance scale of quantum fluctuations that determine the magnitude of the Casimir effect is not the microscopic classical electron radius, as in Pauli's original estimate, much less the even more microscopic Planck length $L_{p l}$, but rather the relatively macroscopic distance $d$ between the conducting boundary surfaces. The resulting subtracted energy density of the vacuum between the conductors is

$$
\rho_{v}=-\frac{\pi^{2}}{720} \frac{\hbar c}{d^{4}}
$$

This energy density is of the opposite sign as (1.4), leading to an attractive force per unit area between the plates of $0.013 \mathrm{dyne} / \mathrm{cm}^{2}(\mu \mathrm{m} / d)^{4}$, a value which is both independent of the ultraviolet cutoff $L_{m i n}^{-1}$, and the microscopic details of the atomic constituents of the conductors. This is a clear indication, confirmed by experiment, that the measurable effects associated with vacuum fluctuations are infrared phenomena, dependent upon macroscopic boundary conditions, which 
have little or nothing to do with the extreme ultraviolet modes or cutoff of the integral in (1.4).

By the Principle of Equivalence, local short distance behavior in a mildly curved spacetime is essentially equivalent to that in flat spacetime. Hence on physical grounds we should not expect the ultraviolet cutoff dependence of (1.4) to affect the universe in the large any more than it affects the force between metallic conductors in the laboratory.

In the case of the Casimir effect a constant zero point energy of the vacuum, no matter how large, does not affect the force between the plates. In the case of cosmology it is usually taken for granted that any effects of boundary conditions can be neglected. It is not obvious then what should play the role of the conducting plates in determining the magnitude of $\rho_{v}$ in the universe, and the magnitude of any effect of quantum zero point energy on the curvature of space has remained unclear from Pauli's original estimate down to the present. In recent years this has evolved from a question of fundamental importance in theoretical physics to a central one of observational cosmology as well. Observations of type Ia supernovae at moderately large redshifts $(z \sim 0.5$ to 1$)$ have led to the conclusion that the Hubble expansion of the universe is accelerating [3]. This is consistent also with microwave background measurements [4]. According to Einstein's equations accelerated expansion is possible if and only if the energy density and pressure of the dominant component of the universe satisfy the inequality,

$$
\rho+3 p=\rho(1+3 w)<0
$$

A vacuum energy with $\rho>0$ and $w \equiv p_{v} / \rho_{v}=-1$ leads to an accelerated expansion, a kind of "repulsive" gravity in which the relativistic effects of a negative pressure can overcome a positive energy density in (1.6). Taken at face value, the observations imply that some $74 \%$ of the energy in the universe is of this hitherto undetected $w=-1$ dark variety [3, 4]. This leads to a non-zero inferred cosmological term in Einstein's equations of

$$
\Lambda_{\text {meas }} \simeq(0.74) \frac{3 H_{0}^{2}}{c^{2}} \simeq 1.4 \times 10^{-56} \mathrm{~cm}^{-2} \simeq 3.6 \times 10^{-122} \frac{c^{3}}{\hbar G} .
$$

Here $H_{0}$ is the present value of the Hubble parameter, approximately $73 \mathrm{~km} / \mathrm{sec} / \mathrm{Mpc} \simeq 2.4 \times$ $10^{-18} \mathrm{sec}^{-1}$. Thus the value of the cosmological dark energy inferred from the SN Ia data in terms of Planck units, $L_{\mathrm{pl}}^{-2}=\frac{c^{3}}{\hbar G}$, gives the dimensionless number in 1.3 the extremely small but finite value,

$$
\lambda \simeq 3.6 \times 10^{-122} .
$$


Explaining the value of this smallest number in all of physics is the basic form of the cosmological constant problem.

As we have already noted, if the universe were purely classical, $L_{p l}$ would vanish and $\Lambda$, like the overall size or total age of the universe, could take on any value whatsoever without any technical problem of naturalness. Likewise as the Casimir effect makes clear, if $G=0$ and there are also no boundary effects to be concerned with, then the cutoff dependent zero point energy of flat space (1.4) could simply be subtracted, with no observable consequences. A naturalness problem arises only when the effects of quantum zero point energy on the large scale curvature of spacetime are considered. This is a problem of the gravitational energy of the quantum vacuum or ground state of the system at macroscopic distance scales, very much greater than $L_{p l}$, when both $\hbar \neq 0$ and $G \neq 0$.

\section{EFFECTIVE FIELD THEORY AND ANOMALIES}

The treatment of quantum effects at distances much larger than any ultraviolet cutoff is precisely the context in which effective field theory (EFT) techniques should be applicable. This means that we assume that we do not need to know every detail of physics at extremely short distance scales of $10^{-33} \mathrm{~cm}$ or even $10^{-13} \mathrm{~cm}$ in order to discuss cosmology at $10^{28} \mathrm{~cm}$ scales. In EFT one assumes some organizing principle or symmetry of low energy dynamics, expresses degrees of freedom in terms of local fields having well-defined covariant transformation properties under the symmetry, and expands the effective action in local invariants of increasing number of derivatives of the fields. The dimensionful parameters multiplying the terms in the action determine the scale at which the derivative expansion is expected to break down.

The organizing principle in gravity is the Principle of Equivalence, i.e. invariance under general coordinate transformations, which greatly restricts the form of any EFT of gravity. In his search for field equations (1.1) for a metric theory with universal matter couplings, which incorporates the Equivalence Principle automatically, but which is no higher than second order in derivatives of the metric, Einstein was using what we would now recognize as EFT reasoning. In an EFT quantum effects and any ultraviolet (UV) divergences they generate at very short distance scales are absorbed into a few, finite low energy effective parameters, such as $G$ and $\Lambda$.

In extending Einstein's classical theory to take account of the quantum properties of matter, the 
classical stress-energy tensor of matter $T_{\nu}^{\mu}$ becomes a quantum operator, with an expectation value $\left\langle T_{\nu}^{\mu}\right\rangle$. In this semi-classical theory with both $\hbar$ and $G$ different from zero, quantum zero-point and vacuum energy effects first appear, while the spacetime geometry can still be treated classically. This is clearly an approximation to a more exact treatment, which can be formally justified by taking the number $N$ of matter degrees of freedom to infinity, and in quantum states which are sharply peaked about their mean value.

Since the expectation value $\left\langle T_{\nu}^{\mu}\right\rangle$ suffers from the quartic divergence 1.4, a regularization and renormalization procedure is necessary in order to define the semi-classical EFT. General coordinate invariance requires a more careful renormalization procedure than the simple subtraction of (1.4) which suffices for the original Casimir calculations in flat space. The UV divergent terms of the stress tensor contain subleading quadratic and logarithmic dependence upon the cutoff $L_{\min }$ which must be isolated and removed in a way consistent with the Equivalence Principle to extract physical effects correctly. These more general renormalization procedures, involving e.g. proper time, covariant point splitting or dimensional regularization have been developed in the context of quantum field theory in curved spacetime [5]. The non-renormalizability of the classical Einstein theory poses no particular obstacle for this semi-classical EFT approach. It requires only that certain additional terms be added to the effective action to take account of UV divergences which are not of the form of a renormalization of $G$ or $\Lambda$. One such set of terms that arise from a consistent covariant renormalization scheme are those associated with the trace anomaly of $\left\langle T_{\nu}^{\mu}\right\rangle$. The result of the renormalization program for quantum fields and their vacuum energy in curved space is that General Relativity can be viewed as a low energy quantum EFT of gravity, provided that the classical Einstein-Hilbert classical action is augmented by the additional terms required by the trace anomaly when $\hbar \neq 0$.

The essential physical assumption in any EFT approach is the hypothesis of decoupling, namely that low energy physics is independent of very short distance degrees of freedom and the details of their interactions. All of the effects of these short distance degrees of freedom are subsumed into a few phenomenological coefficients of the infrared relevant terms of the EFT. Notice that this will not be the case if the low energy $\Lambda$ relevant for dark energy and cosmology depends upon the quantum zero point energies of all fields up to some UV cutoff, as in (1.4). Taken seriously this would indicate quartic power sensitivity of extreme infrared physics to the ultraviolet cutoff. In addition to violating any intuitive notion of decoupling, this is clearly not how the Casimir effect 
works. The hierarchy between the scale of electroweak symmetry breaking in the Standard Model compared to the Planck scale also suggests that low energy physics does not have even quadratic power law sensitivity to the extreme UV cutoff scale $L_{P l}$.

Power law sensitivity to ultraviolet cutoffs may well be an artificial problem of a poor regularization technique, since for example it does not occur in dimensional regularization. On the other hand logarithmic scale sensitivity is the basis of renormalization group analyses, and in the case of the Standard Model has been verified experimentally [6]. The argument of a logarithm necessarily involves the ratio of a UV scale to an IR scale. This distinguishes logarithms from simple additive UV contributions to dimensionful quantities such as the Higgs mass, or the cosomological vacuum energy in (1.4). Logarithmic corrections to classical gravity arises from the conformal or trace anomaly [5, 7].

Anomalies violate strict decoupling of UV from IR degrees of freedom in the sense that the effective action that describes them is necessarily non-local in terms of the original local field degrees of freedom. Because it is non-local in terms of the original fields, the usual EFT approach of expanding in local invariants with higher numbers of derivatives of those fields will miss the anomaly. Instead the non-local effective action of the anomaly must be added explicitly to the local EFT action. Alternatively, an anomaly generally implies massless poles signifying additional massless degrees of freedom which do not decouple, and these new degrees of freedom need to be added to the action to complete the low energy effective theory.

\section{THE AXIAL ANOMALY AND ITS MASSLESS POLE}

The best known example of a quantum anomaly is the chiral or axial anomaly of QED, [8] also present in QCD. Despite this there are some features of the QED axial anomaly that remain

somewhat underappreciated, which are directly relevant to the gravity case. Therefore it is well to review the IR features of the QED axial anomaly before proceeding to gravitational applications.

In QED the Dirac equation

$$
-i \gamma^{\mu}\left(\partial_{\mu}-i e A_{\mu}\right) \psi+m \psi=0
$$

implies that the vector current $J^{\mu}=\bar{\psi} \gamma^{\mu} \psi$ is conserved:

$$
\partial_{\mu} J^{\mu}=0
$$


The axial current $J_{5}^{\mu}=\bar{\psi} \gamma^{\mu} \gamma^{5} \psi\left(\right.$ with $\gamma^{5} \equiv i \gamma^{0} \gamma^{1} \gamma^{2} \gamma^{3}$ ) apparently obeys

$$
\partial_{\mu} J_{5}^{\mu}=2 i m \bar{\psi} \gamma^{5} \psi \quad \text { (classically) }
$$

In the limit of vanishing fermion mass $m \rightarrow 0$, the classical Lagrangian has a $U_{c h}(1)$ global symmetry under $\psi \rightarrow e^{i \alpha \gamma^{5}} \psi$, in addition to $U(1)$ local gauge invariance, and $J_{5}^{\mu}$ is the Noether current corresponding to this chiral symmetry. As is well known, both symmetries cannot be maintained simultaneously at the quantum level [8]. Let us denote by $\left\langle J_{5}^{\mu}(z)\right\rangle_{A}$ the expectation value of the chiral current in the presence of a background electromagnetic potential $A_{\mu}$. Enforcing $U(1)$ gauge invariance 3.2 ) on the full quantum theory leads necessarily to a finite axial current anomaly,

$$
\left.\partial_{\mu}\left\langle J_{5}^{\mu}\right\rangle_{A}\right|_{m=0}=\frac{e^{2}}{16 \pi^{2}} \epsilon^{\mu \nu \rho \sigma} F_{\mu \nu} F_{\rho \sigma}=\frac{e^{2}}{2 \pi^{2}} \vec{E} \cdot \vec{B}
$$

in an external electromagnetic field. Varying this expression twice with respect to the external $A$ field and Fourier transforming, we see that the anomaly must appear in the amplitude,

$$
\begin{aligned}
& \Gamma^{\mu \alpha \beta}(p, q) \equiv-\left.i \int d^{4} x \int d^{4} y e^{i p \cdot x+i q \cdot y} \frac{\delta^{2}\left\langle J_{5}^{\mu}(0)\right\rangle_{A}}{\delta A_{\alpha}(x) \delta A_{\beta}(y)}\right|_{A=0} \\
& =\left.i e^{2} \int d^{4} x \int d^{4} y e^{i p \cdot x+i q \cdot y}\left\langle\mathcal{T} J_{5}^{\mu}(0) J^{\alpha}(x) J^{\beta}(y)\right\rangle\right|_{A=0} .
\end{aligned}
$$

At the lowest one-loop order it is given by a triangle diagram with the axial current $J_{5}^{\mu}$ at one vertex with four-momentum $k^{\mu}$, and the vector currents $J^{\alpha}$ and $J^{\beta}$ at the other two vertices, coupling to photons with four-momenta $p^{\mu}$ and $q^{\mu}$ respectively. Momentum conservation requires $k^{\mu}=p^{\mu}+q^{\mu}$.

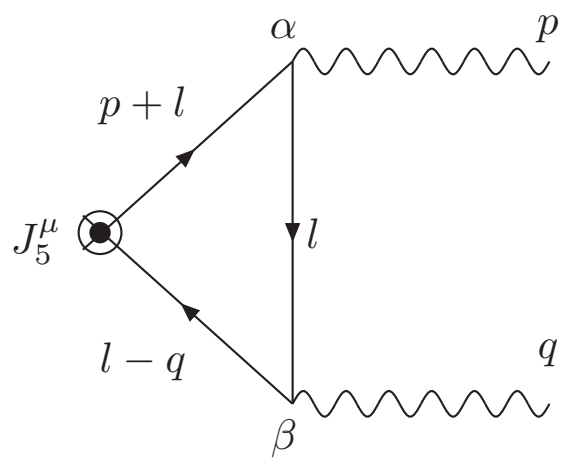

FIG. 1: The Axial Anomaly Triangle Diagram. The momentum integration variable is $l$.

The anomaly may be regarded as a clash of symmetries. Either but not both of the two classically valid relations $(3.2)$ and $(3.3)$ can be maintained at the quantum level. There are various ways to see 
this, but intuitively, defining the quantum amplitude (3.5) at one-loop order introduces implicitly or explicitly an additional scale into the amplitude, which violates naive identities dependent upon global scale and chiral invariance. Although this is usually presented as a UV scale, necessary to regulate the triangle amplitude, in fact it is not difficult to show that the axial anomaly (3.4) is determined by its UV finite, i.e. cutoff independent parts, together with Lorentz invariance, vector current conservation (3.2), and Bose symmetry of exchanging the two external photon lines [9 11]. Thus, the anomaly can be equally well thought of as due to the sensitivity of the amplitude (3.5) to an infrared cutoff at large distances when $m=0$ and $k=p+q=0$.

Consistent with this infrared interpretation of the axial anomaly, one can demonstrate explicitly the existence of a massless pole in the amplitude (3.5), and in the matrix element of the axial current between the vacuum and the physical two-photon state, $|p, q\rangle$, giving [1]

$$
\begin{gathered}
\left\langle 0\left|J_{5}^{\mu}(0)\right| p, q\right\rangle=\left.i \Gamma^{\mu \alpha \beta}(p, q) \tilde{A}_{\alpha}(p) \tilde{A}_{\beta}(q)\right|_{m^{2}=p^{2}=q^{2}=0} \\
=\frac{i e^{2}}{2 \pi^{2} k^{2}} k^{\mu} \epsilon^{\alpha \beta \rho \sigma} p_{\rho} q_{\sigma} \tilde{A}_{\alpha}(p) \tilde{A}_{\beta}(q),
\end{gathered}
$$

When contracted with $k_{\mu}$ the pole cancels and we recover (3.4) in momentum space. The existence of the pole at $k^{2}=0$ in a physical amplitude 3.6 implies the existence of a propagating massless pseudoscalar $0^{-}$intermediate state, with low energy long range effects. The discontinuity of the amplitude contains a $\delta$ function in the corresponding spectral representation, the signal of a massless intermediate state [10, 11]. This new state appears in the two-particle correlations of massless fermions moving collinearly at the speed of light, and are a genuine quantum effect. The effective action for the anomaly is non-local in terms of the original fields but can be rendered local by the introduction of two new massless pseudoscalar fields whose propagator gives rise to the $k^{-2}$ pole in (3.6).

In real QED these infrared effects are suppressed by the non-zero physical electron mass $m>0$, and the additional fact that macroscopic chirality violating sources for $J_{5}^{\mu}$ which would be sensitive to the anomaly are difficult to create. In QCD the situation is complicated by the strong interactions in the infrared and chiral symmetry breaking. The neutral member of the isotriplet of pseudoscalar Goldstone bosons in the low energy EFT is the $\pi^{0}$, whose decay to two photons, $\pi^{0} \rightarrow 2 \gamma$ is correctly given by the triangle amplitude [12]. In fact, it was the experimental agreement between the measured decay rate to that predicted by the axial anomaly computed in the UV theory of 3 colors of fractionally charged quarks that gave one of the strongest early confirmations of QCD. 
It is the fact that the anomaly may be computed in the UV theory but gives rise to a low energy amplitude, $\pi^{0} \rightarrow 2 \gamma$ that led to the principle of anomaly matching [13].

The apparent massless pseudoscalar anomaly pole of in the isosinglet channel in the chiral limit of QCD is even more interesting. This pole mixes with the psuedoscalar axial gluon density $Q(x)=G_{\mu \nu}^{a}(x) \tilde{G}^{a \mu \nu}(x)$, and gives rise to a non-vanishing susceptibility of axial gluon densities,

$$
\chi\left(k^{2}\right)=\int d^{4} x e^{i k \cdot x}\langle Q(x) Q(0)\rangle,
$$

as $k^{2} \rightarrow 0$, despite the fact that $Q$ is a total derivative and therefore one would naively expect $\chi\left(k^{2}\right)$ to be proportional to $k^{2}$ and vanish in this limit. The fact that the susceptibility $\chi(0)$ is non-vanishing is a direct effect of the massless anomaly pole [14. The degree of freedom this infrared pole represents combines with a non-dynamical but gauge invariant $Q^{2}$ term in the effective action of QCD to yield finally one propagating massive isosinglet psuedoscalar state which can be identified with the $\eta^{\prime}$ meson, solving the $U(1)$ problem in QCD [14]. Thus there is no doubt that the pseudoscalar $0^{-}$state which appears in the anomaly channel in perturbation theory is physical and propagating in the final S-matrix of the theory, but it becomes massive by a topological variant of the Higgs mechanism [15].

The lesson to be taken away from this QCD example is that anomalies are a unique window which the low energy EFT provides to short distance physics. As such the anomalous Ward identities and the long distance effects they generate must be taken into account by explicitly adding the IR relevant terms they induce in the low energy effective action [12].

\section{THE TRACE ANOMALY IN TWO DIMENSIONS}

Consider next gravity in $D=2$ dimensions. The local action,

$$
S_{c l}[g]=\int d^{2} x \sqrt{-g}(\gamma R-2 \Lambda)
$$

actually contains no local degrees of freedom at all, since all metrics in $D=2$ are locally conformally flat, and hence may be expressed in the form

$$
g_{\mu \nu}=\exp (2 \sigma) \bar{g}_{\mu \nu}
$$

for some $\sigma(x)$ and a fixed metric $\bar{g}_{\mu \nu}$ which may be taken to be flat. Owing to the identity,

$$
R \sqrt{-g}=\bar{R} \sqrt{-\bar{g}}-2 \sqrt{-\bar{g}} \square \sigma, \quad(D=2)
$$


the $\sigma$ dependence of the Einstein-Hilbert term in (4.1) is a total derivative and gives no metric variation. Hence the theory described by the local action (4.1) has no local dynamical degrees of freedom at all.

When massless conformal matter is coupled to the geometry, this situation changes due to the conformal trace anomaly, [5]

$$
\left\langle T_{\mu}^{\mu}\right\rangle=\frac{N}{24 \pi} R, \quad(D=2)
$$

where $N=N_{S}+N_{F}$ is the total number of massless fields, either scalar $\left(N_{S}\right)$ or fermionic $\left(N_{F}\right)$. It is not difficult to show that the amplitude $\int d^{2} x e^{i k \cdot x}\left\langle T^{\mu \nu}(x) T^{\alpha \beta}(0)\right\rangle$ at one-loop order develops a (gauge invariant) pole at $k^{2}=0$ when the mass of the quantum field vanishes, whose residue is just proportional to the coefficient of the anomaly (4.4] [16]. Accordingly, no local coordinate invariant action exists whose metric variation leads to 4.4 .

A non-local action corresponding to (4.4) can easily be found by using the relation (4.3) in (4.4), which implies that the conformal variation, $\delta \Gamma / \delta \sigma=\sqrt{-g}\left\langle T_{\mu}^{\mu}\right\rangle$ of the effective action $\Gamma$ reproducing the anomaly is linear in $\sigma$. Hence this Wess-Zumino effective action [17] in two dimensions is

$$
\Gamma_{W Z}^{(2)}[\bar{g} ; \sigma]=\frac{N}{24 \pi} \int d^{2} x \sqrt{-\bar{g}}(-\sigma \bar{\square} \sigma+\bar{R} \sigma)
$$

By solving 4.3 for $\sigma$ it is now straightforward to find a non-local scalar functional $S_{\text {anom }}[g]$ of the full metric in 4.2 such that

$$
\Gamma_{W Z}^{(2)}[\bar{g} ; \sigma]=S_{\text {anom }}^{(2)}\left[g=e^{2 \sigma} \bar{g}\right]-S_{\text {anom }}^{(2)}[\bar{g}]
$$

upon also using the fact that $\sqrt{-g} \square=\sqrt{-\bar{g}} \bar{\square}$ is conformally invariant in two dimensions. In this way we find

$$
S_{\text {anom }}^{(2)}[g]=-\frac{N}{96 \pi} \int d^{2} x \sqrt{-g} \int d^{2} x^{\prime} \sqrt{-g^{\prime}} R(x) \square^{-1}\left(x, x^{\prime}\right) R\left(x^{\prime}\right),
$$

with $\square^{-1}\left(x, x^{\prime}\right)$ denoting the Green's function inverse of the scalar differential operator $\square$. The parameter $N$ is replaced by $N-25$ if account is taken of the contribution to the anomaly coefficient of the metric fluctuations themselves in addition to those of the $N$ matter fields.

The anomalous effective action 4.7 is a scalar under coordinate transformations and therefore fully covariant and geometric in character, as required by the Equivalence Principle. However since it involves the Green's function $\square^{-1}\left(x, x^{\prime}\right)$, which requires boundary conditions for its unique 
specification, it is quite non-local, and dependent upon more than just the local curvature invariants of spacetime. In this important respect it is quite different from the classical action (4.1), and describes rather different physics. In order to expose that physics it is most convenient to recast the non-local and non-single valued functional of the metric, $S_{a n o m}^{(2)}$ into a local form by introducing a scalar auxiliary field $\varphi$ satisfying [19]

$$
-\square \varphi=R .
$$

Then one may check that varying

$$
S_{\text {anom }}^{(2)}[g ; \varphi] \equiv-\frac{N}{96 \pi} \int d^{2} x \sqrt{-g}\left(g^{\mu \nu} \nabla_{\mu} \varphi \nabla_{\nu} \varphi-2 R \varphi\right)
$$

with respect to $\varphi$ gives the eq. of motion (4.8) for the auxiliary field, which when solved formally by $\varphi=-\square^{-1} R$ and substituted back into $S_{\text {anom }}^{(2)}[g ; \varphi]$ returns the non-local form of the anomalous action (4.7), up to a surface term. The non-local information in addition to the local geometry which was previously contained in the specification of the Green's function $\square^{-1}\left(x, x^{\prime}\right)$ now resides in the local auxiliary field $\varphi(x)$, and the freedom to add to it homogeneous solutions of (4.8).

In the local form (4.9), we see that a new local scalar degree of freedom has appeared in the form of the auxiliary field $\varphi$, which was not present in the original classical action (4.1). This field is associated with the scalar conformal deformations of the metric, which now fluctuates freely thanks to the kinetic term in (4.9), whereas the metric was constrained in 4.1). The massless pole in the intermediate state of $\int d^{2} x e^{i k \cdot x}\left\langle T^{\mu \nu}(x) T^{\alpha \beta}(0)\right\rangle$ is exactly the $\varphi$ propagator. Its fluctuations lead to the gravitational "dressing" of the critical exponents of conformal matter in a gravitational background [18].

Since critical exponents are characteristic of long range fluctuations in a second order phase transition, this shows that the effective action of the anomaly $(4.9)$ is definitely a relevant operator in the infrared. The anomalous action is also responsible for the infrared running of $\Lambda$. In other words, the bare parameter of the classical action is renormalized by the quantum fluctuations of the $\varphi$ field, and becomes scale dependent. For all of these reasons the action (4.9) and the additional scalar degree of freedom contained in it must be added to the classical action (4.1), to get a complete low energy EFT of two-dimensional gravity. 


\section{MASSLESS SCALAR DEGREES OF FREEDOM IN $4 D$ GRAVITY}

With the examples of the QED/QCD axial anomaly and the conformal anomaly in two dimensions, we consider finally the conformal or trace anomaly in $D=4$ dimensions,

$$
\left\langle T_{\mu}^{\mu}\right\rangle=b F+b^{\prime}\left(E-\frac{2}{3} \square R\right)+b^{\prime \prime} \square R+\sum_{i} c_{i} H_{i},
$$

where

$$
\begin{aligned}
& E \equiv{ }^{*} R_{\mu \nu \alpha \beta}{ }^{*} R^{\mu \nu \alpha \beta}=R_{\mu \nu \alpha \beta} R^{\mu \nu \alpha \beta}-4 R_{\mu \nu} R^{\mu \nu}+R^{2} \\
& F \equiv C_{\mu \nu \alpha \beta} C^{\mu \nu \alpha \beta}=R_{\mu \nu \alpha \beta} R^{\mu \nu \alpha \beta}-2 R_{\mu \nu} R^{\mu \nu}+\frac{R^{2}}{3} .
\end{aligned}
$$

and $H_{i}$ denotes any number of dimension 4 conformally invariant scalars constructed from the gauge fields externally coupled to the matter in question. For example in QED in flat space with $E=F=0$, with massless fermions coupled to electromagnetism, $H=F_{\mu \nu} F^{\mu \nu}$ and one finds

$$
\left.\left\langle T_{\mu}^{\mu}\right\rangle_{A}\right|_{m=0, \text { flat }}=c H=-\frac{e^{2}}{24 \pi^{2}} F_{\mu \nu} F^{\mu \nu},
$$

in complete analogy with (3.4). The coefficients $b$ and $b^{\prime}$ in (5.1), like the coefficient in (3.4) do not depend on any ultraviolet short distance cutoff, but instead are determined only by the number and spin of massless fields [5, 7],

$$
\begin{aligned}
b & =\frac{\hbar}{120(4 \pi)^{2}}\left(N_{S}+6 N_{F}+12 N_{V}\right), \\
b^{\prime} & =-\frac{\hbar}{360(4 \pi)^{2}}\left(N_{S}+\frac{11}{2} N_{F}+62 N_{V}\right),
\end{aligned}
$$

with $\left(N_{S}, N_{F}, N_{V}\right)$ the number of fields of spin $\left(0, \frac{1}{2}, 1\right)$ respectively.

For the flat space trace anomaly in an external electromagnetic field $(5.3)$ one can perform a full analysis of the one-loop triangle contribution to the amplitude,

$$
\Gamma^{\mu \nu \alpha \beta}(p, q)=\int d^{4} x \int d^{4} y e^{i p \cdot x+i q \cdot y}=\left.\frac{\delta^{2}\left\langle T^{\mu \nu}(0)\right\rangle_{A}}{\delta A_{\alpha}(x) \delta A_{\beta}(y)}\right|_{A=0},
$$

analogous to (3.5). This triangle amplitude analogous to that in Fig. 1 (with $J_{5}^{\mu}$ replaced by $T^{\mu \nu}$ ) also develops a pole at $k^{2}=0$ when the combined limits $p^{2}=q^{2}=m^{2}=0$ are taken. The $0^{+}$ scalar pole survives in matrix elements of the stress-energy tensor of massless fermions to physical two-photon states, analogous to (3.6). The residue of the pole is proportional to the coefficient of the anomaly. The discontinuity of the $\langle T J J\rangle$ triangle diagram also exhibits a $\delta$ function in 
the intermediate two-electron state with total spin zero, which signifies that a new massless scalar degree of freedom with gravitational coupling is required by the anomaly [11].

The effective action of the massless degree(s) of freedom is again non-local in terms of the original local metric and electromagnetic field strengths. However it can be rewritten in a completely local form by the introduction of the two local scalar fields whose quanta are responsible for the massless poles in anomalous amplitudes such as the $\langle T J J\rangle$ and $\langle T T T\rangle$ triangle diagrams. By following steps analogous to those in the two dimensional case of the previous section, explicitly integrating the anomaly eq. (5.1) and introducing two auxiliary fields $\varphi$ and $\psi$ to account for the two independent invariants $b^{\prime} E$ and $b F$ in the trace anomaly (5.1) one finds [19 22]

$$
\begin{aligned}
& S_{\text {anom }}[g ; \varphi, \psi]=\frac{b^{\prime}}{2} \int d^{4} x \sqrt{-g}\left\{-\varphi \Delta_{4} \varphi+\left(E-\frac{2}{3} \square R\right) \varphi\right\} \\
& +\frac{b}{2} \int d^{4} x \sqrt{-g}\left\{-2 \varphi \Delta_{4} \psi+\left(F+\frac{c}{b} H\right) \varphi+\left(E-\frac{2}{3} \square R\right) \psi\right\}
\end{aligned}
$$

where in QED, $c=-e^{2} / 24 \pi^{2}, H=F_{\mu \nu} F^{\mu \nu}$ and in general,

$$
\Delta_{4} \equiv \square^{2}+2 R^{\mu \nu} \nabla_{\mu} \nabla_{\nu}-\frac{2}{3} R \square+\frac{1}{3}\left(\nabla^{\mu} R\right) \nabla_{\mu} .
$$

By variation of 5.6 the auxiliary scalar fields satisfy the linear fourth order eqs. of motion,

$$
\begin{aligned}
& \Delta_{4} \varphi=\frac{1}{2} E-\frac{1}{3} \square R, \\
& \Delta_{4} \psi=\frac{1}{2} F+\frac{c}{2 b} H .
\end{aligned}
$$

The effective action (5.6) analogous to 4.9 in $D=2$ generates all the anomalous amplitudes, by successive variations with respect to background metric and/or external gauge potentials, including diagrams with multiple stress-energy tensor insertions, such as $\langle T T T \ldots J J\rangle$ and $\langle T T T \ldots\rangle$.

Since from the free variation of the effective action (5.6) $\varphi$ and $\psi$ obey non-trivial massless wave eqs., they are additional massless scalar degrees of freedom in low energy gravity, over and above the usual transverse, tracefree gravitational waves of the Einstein theory, and can have long range, macroscopic effects. The poles in the amplitude (5.5) survive in low energy scattering processes involving two photons with a gravitational strength [11].

For QED the effects of the trace anomaly $\langle T J J\rangle$ in flat space are again screened at distances greater than $\hbar / m c$ by the finite mass of the electron. However the trace anomalies in $\langle T T T \ldots\rangle$ amplitudes which couple to purely gravitational sources and scattering processes are mediated by 
truly massless particles, such as the photon itself. Unlike the axial case, where non-trivial sources for $J_{5}^{\mu}$ may not be easy to come by, the sources here are gravitational which are omnipresent in the Universe. Since the coupling is through the stress-energy, it is universal but weak. However gravity is unscreened by any other interaction and its effects are cumulative over large distances. The long range nature of massless fluctuations make the scalar degrees of freedom contained in the effective action (5.6) relevant at the even the very largest macroscopic distance scales of cosmology.

\section{DYNAMICAL VACUUM ENERGY}

The example of the axial anomaly, well-tested in QCD, and the logarithmic scaling of the anomaly action (5.6) imply that the full effective action of low energy gravity should be the sum of the classical Einstein-Hilbert action of classical General Relativity together with the effective action (5.6). In this EFT the additional long range scalar modes have some interesting effects.

With a covariant action functional (5.6) one may compute a covariantly conserved stress-energy tensor $[22]$

$$
T_{\mu \nu}^{(\text {anom })} \equiv-\frac{2}{\sqrt{-g}} \frac{\delta S_{a n o m}}{\delta g^{\mu \nu}}
$$

the $b^{\prime}$ term of which gives the tensor,

$$
\begin{aligned}
E_{\mu \nu}= & -\frac{2}{3} \partial_{\mu} \partial_{\nu} \square \varphi-2\left(\partial_{(\mu} \varphi\right) \partial_{\nu)} \square \varphi+2\left(\partial_{\mu} \partial_{\nu} \varphi\right) \square \varphi+\frac{2}{3}\left(\partial_{\alpha} \varphi\right)\left(\partial^{\alpha} \partial_{\mu} \partial_{\nu} \varphi\right) \\
& -\frac{4}{3}\left(\partial_{\mu} \partial_{\alpha} \varphi\right)\left(\partial_{\nu} \partial^{\alpha} \varphi\right)+\frac{1}{6} \eta_{\mu \nu}\left\{-3(\square \varphi)^{2}+\square\left[\left(\partial_{\alpha} \varphi\right)\left(\partial^{\alpha} \varphi\right)\right]\right\}
\end{aligned}
$$

in flat space, which is conserved by use of the eqs. of motion (5.8). A particular solution of (5.8a) in flat space with $E=\square R=0$ is

$$
\varphi=a \frac{z^{2}}{d^{2}}
$$

(with the second auxiliary field $\psi=0$ ) which leads to

$$
T_{\mu \nu}^{(\text {anom })}=b^{\prime} E_{\mu \nu}=-\frac{2 b^{\prime} a^{2}}{3 d^{4}}\left(\eta_{\mu \nu}-4 \delta_{\mu}^{z} \delta_{\nu}^{z}\right)=\frac{C}{d^{4}} \operatorname{diag}(-1,1,1,-3) .
$$

This is exactly the form of the Casimir vacuum stress-energy between two infinite parallel conducting plates a distance $d$ apart in (1.5). The constant $a$ and therefore $C=-2 b^{\prime} a^{2} / 3$ depends upon the boundary conditions imposed on the conductors. Notice from $5.4 \mathrm{~b}$ ) that $b^{\prime}<0$ so that 
the constant $C>0$, corresponding to an attractive force between the plates for any real $a$. It is remarkable that the auxiliary field $\varphi$ and its stress tensor, obtained from the local form of quantum anomaly in the trace of the stress-energy tensor in curved space may be regarded through a particular homogeneous solution (6.3) of the classical eqs. (5.8) as responsible for the Casimir stress tensor (6.4) in flat space, where it is tracefree.

Several other examples of the auxiliary fields and stress tensor (6.1) in curved black hole and cosmological spacetimes have now been studied and used to compute vacuum polarization, illuminating their physical meaning [22, 23]. Solving classical differential eqs. for the auxiliary fields in a given fixed spacetime background allows one to survey a great number of physical states of the underlying quantum field theory, taking account the spin of the field through the $b, b^{\prime}$ coefficients (5.4), rather than having to decompose the solutions of the field eqs. for each spin into normal modes, impose boundary conditions on those modes, construct the stress tensor and regularize it and renormalize it in each quantum state. The stress-energy (6.1) is particularly important in the vicinity of black hole and cosmological horizons, which it can dominate even the classical curvature terms, and lead to large quantum vacuum polarization effects there [22, 24].

In addition, when the scalar fields $\varphi, \psi$ in (5.6) are treated as dynamical fields and quantized in their own right, they lead to infrared renormalization and finite volume dependence of the effective cosmological term (1.3). In other words the quantity $\lambda=\hbar G \Lambda / c^{3}$ becomes a dynamical quantity in the EFT, running with IR renormalization scale as every other coupling affected by light degrees of freedom. A one-loop calculation with the auxiliary field propagator given by the inverse of (5.7) gives the volume scaling relation [25]

$$
V \frac{d \lambda}{d V}=4(2 \delta-1) \lambda
$$

with the anomalous dimension,

$$
2 \delta-1=\frac{\sqrt{1-\frac{8}{Q^{2}}}-\sqrt{1-\frac{4}{Q^{2}}}}{1+\sqrt{1-\frac{4}{Q^{2}}}} \leq 0,
$$

and

$$
Q^{2} \equiv-32 \pi^{2} b^{\prime}=\frac{1}{180}\left(N_{S}+\frac{11}{2} N_{F}+62 N_{V}\right)+Q_{g r a v}^{2}
$$

in terms of the anomaly coefficient $b^{\prime}$ in 5.1, and $Q_{\text {grav }}^{2}$, the contribution of graviton fluctuations to the anomaly coefficient (approximately 7.9). The anomalous scaling dimension (6.6) is negative 
for all $Q^{2} \geq 8$. This implies that the dimensionless cosmological term $\lambda$ has an infrared fixed point at zero as the volume $V \rightarrow \infty$. Thus the cosmological term is dynamically driven to zero as $V \rightarrow \infty$ by infrared fluctuations of the conformal part of the metric described by (5.6). There is no fine tuning involved here and no free parameters enter except $Q^{2}$, which is determined by the trace anomaly coefficient $b^{\prime}$ by (6.7). Once $Q^{2}$ is assumed to be positive, then $2 \delta-1$ is negative, and $\lambda$ is driven to zero at large volumes or large distances by the conformal fluctuations of the metric. This identifies a mechanism for the dynamical screening of the vacuum energy at large distances, relying only on the four dimensional quantum physics of the trace anomaly with no additional assumptions.

Thus, the fluctuations of the new scalar degrees of freedom in the effective action (5.6) of the anomaly allow the cosmological "constant" vacuum energy of classical General Relativity to vary dynamically. This is qualitatively similar to the effect of the $\varphi$ conformal degree of freedom in $2 D$ gravity 4.9 at second order critical points [18. The fixed point of $\lambda=0$ is stable to marginal deformations by the Einstein-Hilbert terms, and describes a quantum conformal phase of $4 D$ gravity. To take account of this mechanism and understand the role of the conformal phase where the fluctuations of the new dynamical scalar degrees of freedom are important in a consistent cosmological theory of vacuum dark energy is the remaining task.

\section{LINEAR RESPONSE IN DE SITTER SPACE AND COSMOLOGICAL HORIZON MODES}

To this end we have recently studied the effect of the scalar fluctuations in the linear response of coupled matter-geometry perturbations around de Sitter spacetime, relevant for both inflationary and present day dark energy cosmology [24]. Here only the main results are summarized.

Linear response in gravity means solving the linear eqs. for small perturbations of the metric and matter stress-energy renormalized expectation value $\left\langle T_{\nu}^{\mu}\right\rangle_{R}$ expanded around a self-consistent solution of the semi-classical Einstein equations. A self-consistent solution of these eqs. is de Sitter space,

$$
d s^{2}=-c^{2} d \tau^{2}+a^{2}(\tau)\left(d x^{2}+d y^{2}+d z^{2}\right)=-c^{2} d \tau^{2}+e^{2 H \tau} d \vec{x} \cdot d \vec{x}
$$

with $a(\tau)=e^{H \tau}$ the de Sitter Robertson-Walker (RW) scale factor, and conformal matter fields in their de Sitter invariant Bunch-Davies state [5]. The linear variation of the semi-classical Einstein 
eqs. is:

$$
\delta\left\{R^{\mu}{ }_{\nu}-\frac{R}{2} \delta^{\mu}{ }_{\nu}+\Lambda \delta^{\mu}{ }_{\nu}\right\}=\frac{8 \pi G}{c^{4}} \delta\left\langle T_{\nu}^{\mu}\right\rangle_{R}
$$

The variation on the left side is purely geometrical, obtained by varying the metric from its de Sitter value $g_{\mu \nu}$ given in RW coordinates (7.1) to $g_{\mu \nu}+\delta g_{\mu \nu}$. The variation of the expectation value on the right side contains two kinds of terms. The first kind are also proportional to the metric variation $h_{\mu \nu}=\delta g_{\mu \nu}$ and involve the retarded response function of stress-energy fluctuations, namely $\theta\left(t-t^{\prime}\right)\left\langle\left[T_{\nu}^{\mu}(x), T^{\alpha \beta}\left(x^{\prime}\right)\right]\right\rangle$ integrated over all points $x^{\prime}$ in the causal past of the point $x$. It turns out that this first kind of variation leads to solutions of linear response eqs. (7.2) which have spacetime dependence only on the Planck scale 1.2 . Since this is the ultraviolet cutoff scale at which the semi-classical EFT breaks down, one cannot trust any physical conclusion obtained with this first kind of solution of 7.2 .

The second kind of term in the stress-energy variation $\delta\left\langle T_{\nu}^{\mu}\right\rangle_{R}$ in 7.2 arise from the possibility of varying the quantum state of the field in which the expectation value $\left\langle T_{\nu}^{\mu}\right\rangle_{R}$ is evaluated, independently of the variation of the metric. The quantum state is specified by boundary conditions on the cosmological horizon scale $c / H$, having nothing to do with the microscopic Planck scale $L_{P l}$. The scalar auxiliary field effective action (5.6) and eqs. (5.8) in de Sitter space parametrize additional state dependent contributions to $\delta\left\langle T^{\mu}\right\rangle_{R}$ from the variation of $\delta T_{\mu \nu}^{(\text {anom })}$ of 6.1 . The gauge invariant combination of auxiliary field $\delta \varphi$ and metric perturbation $h_{\tau \tau}$ given by

$$
u=\frac{1}{H^{2}}\left(\frac{\partial^{2}}{\partial \tau^{2}}+H \frac{\partial}{\partial \tau}-\frac{\vec{\nabla}^{2}}{a^{2}}\right) \delta \varphi-2 h_{\tau \tau}
$$

in the gauge $g^{i j} h_{i j}=0=\nabla^{i} h_{i \tau}-\frac{1}{2} h_{\tau \tau}$ satisfies the second order homogeneous eq., [24]

$$
\left(\frac{\partial^{2}}{\partial \tau^{2}}+5 H \frac{\partial}{\partial \tau}+6 H^{2}-\frac{\vec{\nabla}^{2}}{a^{2}}\right) u=0,
$$

the general solution of which is a linear combination of

$$
u_{\vec{k}, \pm}(\tau, \vec{x})=v_{\vec{k}, \pm}(\tau, \vec{x})=\frac{1}{a^{2}} \exp \left( \pm \frac{i k}{H a}+i \vec{k} \cdot \vec{x}\right)
$$

in Fourier space. These modes and those of the same form arising from the second auxiliary field $\psi($ called $v$ ), give rise in the linear response eq. 7.2 to perturbations of the Ricci tensor,

$$
\delta R_{\tau}^{\tau}=-\delta R_{i}^{i}=\frac{\varepsilon^{\prime}}{2 a^{2}} \vec{\nabla}^{2} u-\frac{\varepsilon}{6 a^{2}} \vec{\nabla}^{2} v
$$


with $\delta R=0$ and

$$
\begin{aligned}
\varepsilon & \equiv 32 \pi G H^{2} b, \\
\varepsilon^{\prime} & \equiv-\frac{32 \pi}{3} G H^{2} b^{\prime} .
\end{aligned}
$$

Thus the auxiliary fields of the anomaly action yield non-trivial gauge invariant solutions for the stress tensor and corresponding linearized Ricci tensor perturbations (7.6). Being solutions of (7.4) which itself is independent of the Planck scale, these solutions vary instead on arbitrary scales determined by the wavevector $\vec{k}$, and are therefore genuine low energy modes of the semiclassical effective theory. The Newtonian gravitational constant $G$ and the Planck scale enter (7.6) only through the small coupling parameters $\varepsilon$ and $\varepsilon^{\prime}$ between the auxiliary fields and the metric perturbation. In the limit of either flat space, or arbitrarily weak coupling $G H^{2} \rightarrow 0$ these modes decouple from the metric perturbations at linear order. Thus, there is no problem with possible negative metric modes of the $\varphi, \psi$ fields propagating to infinity and leading to a non-unitary $S$ matrix in flat space. However, in a curved space such as de Sitter space these modes can have physical infrared effects at the horizon scale where they do couple.

The infrared scalar $u$ and $v$ modes are associated with the cosmological horizon scale $c / H$ in de Sitter space and for that reason may be called cosmological horizon modes. To show this connection, one may introduce the static coordinates of de Sitter space, viz.

$$
d s^{2}=-\left(c^{2}-H^{2} r^{2}\right) d t^{2}+\frac{d r^{2}}{1-H^{2} r^{2} / c^{2}}+r^{2}\left(d \theta^{2}+\sin ^{2} \theta d \phi^{2}\right)
$$

related to the RW coordinates $(7.1)$ by the coordinate transformation,

$$
\begin{aligned}
r & =|\vec{x}| e^{H \tau} \\
t & =\tau-\frac{1}{2 H} \ln \left(1-\frac{H^{2}|\vec{x}|^{2}}{c^{2}} e^{2 H \tau}\right) .
\end{aligned}
$$

In these static coordinates the eqs. of motion (7.4) possess the time independent solution,

$$
u=v=\frac{1}{1-H^{2} r^{2} / c^{2}}
$$

which diverge on the cosmological horizon $r=c / H$ centered at the origin. The corresponding stress-energy tensor perturbation in the static frame is

$$
\delta\left\langle T_{r}^{r}\right\rangle_{R}=\delta\left\langle T_{\theta}^{\theta}\right\rangle_{R}=\delta\left\langle T_{\phi}^{\phi}\right\rangle_{R}=-\frac{1}{3} \delta\left\langle T_{t}^{t}\right\rangle_{R}=C \frac{\hbar H^{4} / c^{3}}{\left(1-H^{2} r^{2} / c^{2}\right)^{2}}
$$


with a quadratically divergent value on the cosmological horizon. This form of the stress tensor perturbation is also the form of a finite temperature fluctuation away from the Hawking-de Sitter temperature $T_{H}=\hbar H / 2 \pi k_{B}$ of the Bunch-Davies state in static coordinates [26]. It corresponds therefore to a change of the boundary conditions on the state of the underlying quantum fields on the horizon, with a corresponding change in the vacuum polarization effects of the fields near the horizon.

That these Casimir like effects are dynamical and functions of the boundary conditions is not surprising given our experience with the Casimir effect in flat space. It is also known that the Casimir stress-energy can diverge as a curved surface of a perfect conductor is approached [27]. In electromagnetism we know that perfect conductors do not exist and this mathematical divergence is cut off by the finite conductivity and skin depth of a metallic surface [2]. A diverging stress tensor on the cosmological horizon signals the breakdown of boundary conditions there as well, except that in classical General Relativity the horizon is supposed to be purely a mathematical boundary with no physical stress tensor. Large stresses on the horizon of the form (7.11) suggest that this assumption may be incorrect.

In the semi-classical EFT with $S_{\text {anom }}$ included in the effective action there are additional scalar degrees of freedom in gravity that become important in the vicinity of geometries with horizons and there their fluctuations must be taken into account, just as charged matter fluctuations must be taken into account in an imperfect conductor. The stress-energy (7.11) of these degrees of freedom in the vicinity of the cosmological horizon can cause large backreaction on the classical geometry. Because the horizon is a null surface, the massless propagator pole associated with the anomalous amplitudes as in (3.6) can lead to large quantum correlations in multi-stress tensor amplitudes $\langle T T T \ldots\rangle$ on the horizon as well. Such large amplitudes in fluctuations from the mean $\left\langle T_{\nu}^{\mu}\right\rangle$ is characteristic of a phase transition in which the semi-classical mean field theory breaks down, and where the rigid cosmological constant term of the classical theory can change.

Thus the fluctuations of the scalar degrees of freedom determined by the anomaly may lead to a phase transition to precisely the conformally invariant phase of gravity described by the fixed point $\lambda=0$ of 6.5 in the near vicinity of the horizon. This suggests a rather different cosmological model than the standard one, in which we live inside a kind of "bubble" of vacuum energy condensate, with a preferred origin and a physical surface at the cosmological horizon [25, 28]. At the horizon the quantum fluctuations of the scalar degrees of freedom contained in the anomaly lead to a phase 
transition in which the spacetime condensate $\Lambda$ "melts." The $\Lambda$ condensate will then behave very much like the gluon condensate in the QCD bag model of hadrons.

In the low energy EFT of gravity that takes account of the trace anomaly, the value of the cosmological dark energy in the interior is dynamical and should be fixed by the boundary conditions on the surface at the infrared Hubble scale $c / H$, much as the Casimir effect is, with no regard to the ultraviolet divergent and clearly incorrect estimate of (1.4). The consequences of this dynamical dependence upon infrared boundary conditions of the vacuum energy for cosmology, microwave background anisotropies and non-Gaussianities, and in the gravitational collapse problem are presently under investigation.

\section{References}

[1] W. Pauli (unpublished); N. Straumann, e-print arxiv: gr-qc/0208027;

See also W. Nernst, Verh. Dtsch. Phys. Ges. 18, 83 (1916);

C. P. Enz and A. Thellung, Helv. Phys. Acta 33, 839 (1960);

S. E. Rugh and H. Zinkernagel, Stud. Hist. Philos. Mod. Phys. 33, 663 (2002).

[2] K. A. Milton, The Casimir effect: physical manifestations of zero-point energy (World Scientific, Singapore, 2001);

M. Bordag, Advances In the Casimir Effect (Oxford Univ. Press, Oxford, 2009), and references therein.

[3] A. G. Riess et. al., Astron. J. 116, 1009 (1998); Astron. J. 607665 (2004);

S. Perlmutter et. al., Astrophys. J. 517565 (1999);

J. L. Tonry et. al., Astrophys. J. 594, 1 (2003).

[4] C. L. Bennett et. al, WMAP team, Astrophys. J. 583, 1 (2003); e-print arXiv: 1001.4758 (2010).

[5] N. D. Birrell and P. C. W. Davies, Quantum Fields in Curved Space (Cambridge Univ. Press, Cambridge, 1982), and references therein.

[6] See e.g. P. Achard et. al., L3 Collaboration, Phys. Lett. 623B, 26 (2005).

[7] D. Capper and M. Duff, N. Cimento A23, 173 (1974); Phys. Lett. 53A, 361 (1975);

S. Deser, M. Duff and C. J. Isham, Nucl. Phys. B111, 45 (1976);

M. Duff, Nucl. Phys. B125, 334 (1977); Nucl. Phys. B234, 269 (1984); Class. Quant. Grav. 11, 1387 (1994).

[8] S. L. Adler, Phys. Rev. 177, 2426 (1969);

J. S. Bell and R. Jackiw, N. Cimento A60, 47 (1969). 
[9] A. D. Dolgov and V. I. Zakharov, Nucl. Phys. B27, 525 (1971);

J. Hořejší, Czech. J. Phys. B35, 820 (1985); Phys. Rev. D32, 1029 (1985); J. Phys. G: Nucl. Phys. 12, L7 (1986); Czech. J. Phys. 42, 345 (1992).

[10] J. Hořejší, Czech. J. Phys. 42, 241 (1992).

[11] M. Giannotti and E. Mottola, Phys. Rev. D79, 045014 (2009).

[12] W. A. Bardeen, H. Fritzsch and M. Gell-Mann, in Scale and Conformal Symmetry in Hadron Physics, R. Gatto, ed. (Wiley, New York, 1973);

T. P. Cheng and L. F. Li, Gauge Theory of Elementary Particle Physics (Oxford Univ. Press, Oxford, 1984);

S. Weinberg, The Quantum Theory of Fields, Vol. 2 (Cambridge Univ. Press, Cambridge, 1995).

[13] G. 't Hooft in Recent Developments in Gauge Theories, Procedings, NATO Advanced Study Institute, Cargese, Nato Advanced Study Institutes Series B, Physics, Vol. 59 (Plenum, New York,1980).

[14] G. Veneziano, Nucl. Phys. B159, 213 (1979);

P. Di Vecchia and G. Veneziano, Nucl. Phys. B171, 253 (1980).

[15] A. Aurilia, Y. Takahashi, and P. K. Townsend, Phys. Lett. 95B, 265 (1980).

[16] R. A. Bertlmann and E. Kohlprath, Ann. Phys. 288137 (2001).

[17] J. Wess and B. Zumino, Phys. Lett. 37B 95 (1971).

[18] V. G. Knizhnik, A. M. Polyakov, and A. B. Zamolodchikov, Mod. Phys. Lett. A3 819 (1988);

F. David, Mod. Phys. Lett. A3 1651 (1988).

[19] R. J. Riegert, Phys. Lett. 134B, 56 (1984).

[20] E. S. Fradkin and A. A. Tseytlin, Phys. Lett. 134B, 187 (1984).

[21] I. L. Shapiro and A. G. Jacksenaev, Phys. Lett. 324B, 286 (1994);

R. Balbinot, A. Fabbri, and I. L. Shapiro, Phys. Rev. Lett. 83, 1494 (1999); Nucl. Phys. B559, 301 (1999).

[22] E. Mottola and R. Vaulin, Phys. Rev. D 74, 064004 (2006).

[23] P. R. Anderson, E. Mottola, and R. Vaulin, Phys. Rev. D 76, 124028 (2007).

[24] P. R. Anderson, C. Molina-Pariís and E. Mottola, Phys. Rev. D 80, 084005 (2009).

[25] I. Antoniadis, P. O. Mazur, and E. Mottola, N. Jour. Phys. 911 (2007).

[26] P. R. Anderson, W. A. Hiscock, and D. A. Samuel, Phys. Rev. D 51, 4337 (1995).

[27] D. Deutsch and P. Candelas, Phys. Rev. D 20, 3063 (1979).

[28] P. O. Mazur and E. Mottola, arXiv:gr-qc/0109035 Proc. Nat. Acad. Sci. 101, 9545 (2004). 